\title{
Equivalence between the Lagrangian and Hamiltonian formalism for constrained systems
}

\author{
C. Batlle \\ Departament de Fisica Teorica, Universitat de Barcelona, Barcelona, Spain
}

J. Gomis

Department of Physics, Queens College of the City University of New York, Flushing, New York 113670904 and Department of Physics, City College of the City, University of New York, New York, New York 10031

J. M. Pons

Departament de Fisica Teorica, Universitat de Barcelona, Barcelona, Spain

N. Roman-Roy

Departament de Fisica, Escola Tècnica Superior d'Enginyers Industrials de Barcelona, Universitat Politecnica de Catalunya, Barcelona, Spain

(Received 25 November 1985; accepted for publication 20 August 1986)

The equivalence between the Lagrangian and Hamiltonian formalism is studied for constraint systems. A procedure to construct the Lagrangian constraints from the Hamiltonian constraints is given. Those Hamiltonian constraints that are first class with respect to the Hamiltonian constraints produce Lagrangian constraints that are FL-projectable.

\section{INTRODUCTION}

The current interest in constrained systems was spawned by Dirac ${ }^{1}$ and Bergmann ${ }^{2}$ in their study of the canonical formalism of gravitational fields. Since that time several people contributed to the building of a mechanics for such systems. ${ }^{3}$ In particular the Lagrangian, ${ }^{4}$ Hamiltonian, ${ }^{4,5}$ Hamilton-Jacobi, ${ }^{6}$ and geometrical formalisms ${ }^{7}$ have been studied. For a time this field of research had little more than mathematical interest, but now with the increasing interest in gauge theories (any theory with gauge transformations is a theory of constrained systems), more people are beginning to use this formalism at the classical and quantum level.

On the other hand, constrained systems with a finite number of degrees of freedom have been used to construct an $\mathrm{N}$-body relativistic mechanics of direct interactions ${ }^{8}$ whose corresponding quantum mechanics, ${ }^{9}$ which is multitemporal, is related to the Bethe-Salpeter equation.

Despite increasing interest, the mechanics of these systems is not as elaborate as the corresponding mechanics for unconstrained systems. For example, the equivalence between the Lagrangian and Hamiltonian formalism has not been definitely established. ${ }^{10-12}$

In this paper we give an explicit and complete proof of this equivalence. We construct an implicit inverse relation between velocities and momenta, i.e., the inverse Legendre transformation. Using that we deduce the Hamilton-Dirac equations from Euler-Lagrange equations. Neither is a set of normal differential equations, therefore the uniqueness and existence theorem cannot be applied. This means that, at most, we will only have solutions in a submanifold of the respective spaces and in general these solutions will not be unique.

A careful analysis shows that given a solution of the Euler-Lagrange equations we can construct a solution of the Hamilton-Dirac equations and vice versa. Next we look for the appropriate submanifold of the tangent bundle $(T Q)$ and a submanifold of the cotangent bundle $\left(T^{*} Q\right)$ where the solutions exist. These submanifolds are constructed through an iterative procedure. In a given local chart they are characterized by a set of functions that are called constraints.

The Hamiltonian formalism as developed in this paper differs from the usual development. ${ }^{1,2}$ The first class primary constraints play a privileged role. Other constraints are either first or second class with respect to them. These constraints that are first class with respect to the primary first class constraints can be associated with Lagrangian constraints that are FL-projectable (or weakly FL-projectable). Those that are second class in the Hamiltonian formalism have associated non-FL-projectable Lagrangian constraints. It is also shown that all constraints other that the primary constraints have either a symmetric or antisymmetric Poisson bracket (PB) structure with the first class primary constraints.

The paper is organized as follows. In Sec. II we show that if we have a solution of the Euler-Lagrange equations we can construct from it a solution of the Hamilton-Dirac equations and vice versa. In Sec. III we develop an algorithm for the determination of the Hamiltonian constraints. In Sec. IV we develop an analogous algorithm for the Lagrangian constraints and we relate the Lagrangian and Hamiltonian constraints.

\section{THE EQUIVALENCE THEOREMS}

We consider an $N$-dimensional configuration space $Q$ and a function $L$, the Lagrangian, defined in its tangent bundle $T Q$. The Euler-Lagrange equations

$$
\frac{d}{d t} \frac{\partial L}{\partial \dot{q}^{j}}-\frac{\partial L}{\partial q^{j}}=0 ; j=1, \ldots, n,
$$

can be written in the normal form of ordinary second-order differential equations (SODE) only when the Hessian ma- 
trix $W_{i j} \equiv \partial^{2} L / \partial \dot{q}^{i} \partial \dot{q}^{j}$ is regular.

If the Hessian matrix is singular, neither the existence nor uniqueness theorems for SODE holds. This means that the possible solutions of (2.2) lie in a submanifold of $T Q$ and given a point of that submanifold we can have more than one solution passing through that point. We shall assume in the following that the rank of the Hessian matrix $W$ is constant in all $T Q$ and is $n-m_{1}$. If this is not the case, our considerations will only hold in an open region of $T Q$ where this condition is satisfied.

\section{A. The map FL}

The fiber derivative of the Lagrangian is the application (FL) of the tangent bundle on the cotangent bundle $T^{*} Q$

$$
\text { FL: } T Q \rightarrow T^{*} Q \text {, }
$$

given by $\mathrm{FL}(q, \dot{q})=(q, p)$, where

$$
p_{i}=\frac{\partial L}{\partial \dot{q}^{i}} \equiv \mathscr{P}_{i}(q, \dot{q}) \quad i=1, \ldots, n .
$$

We shall also assume that $\mathrm{FL}(T Q)=M_{0} \subset T^{*} Q$ is a submanifold of $T^{*} Q$, locally defined by the constraints

$$
\Phi_{\mu}^{(0)}(q, p)=0, \quad \mu=1,2, \ldots, m_{1},
$$

which are the primary constraints.

We also assume

$$
\operatorname{rank}\left|\frac{\partial \Phi_{\mu}^{(0)}}{\partial p_{j}}\right|=m_{1} \text {. }
$$

This condition excludes ineffective constraints at this level. In the following we will disregard Lagrangians that have ineffective constraints at any level.

The primary Hamiltonian constraints (2.3) are identified at the Lagrangian level, i.e.,

$$
\Phi_{\mu}^{(0)}(q, \mathscr{P}(q, \dot{q})) \equiv 0,
$$

or equivalently

$$
\mathrm{FL}^{*} \boldsymbol{\Phi}_{\mu}^{(0)} \equiv 0 \text {, }
$$

where $\mathrm{FL}^{*}$ is the pullback application. From (2.5) we deduce

$$
\frac{\partial \Phi_{\mu}^{(0)}}{\partial p_{i}}(q, \mathscr{P}(q, \dot{q})) \frac{\partial \mathscr{P}_{i}}{\partial \dot{q}_{j}}=0,
$$

and since $\partial \mathscr{P}_{i} / \partial \dot{q}^{j}$ is the Hessian matrix element $W_{i j}$, we have a basis for the null vectors of $W$ :

$$
\gamma_{\mu}^{i}=\mathrm{FL}^{*} \frac{\partial \Phi_{\mu}^{(0)}}{\partial p^{i}} \quad \mu=1, \ldots, m_{1}, \quad i=1, \ldots, n .
$$

A basis for the kernel of differential application $\mathrm{FL}^{*}$ can be written in terms of (2.7):

$$
\Gamma_{\mu}=\gamma_{\mu}^{i}(q, \dot{q}) \frac{\partial}{\partial \dot{q}_{i}}
$$

A function $f \in \Lambda^{\circ}(\mathrm{TQ})$ is FL-projectable if there exists some function $g \in \Lambda^{0}\left(T^{*} Q\right)$ such that $f=\mathrm{FL}^{*} g$. The necessary and sufficient condition for $f \in \Lambda^{0}(\mathrm{TQ})$ to be FL-projectable is that ${ }^{7,10}$

$$
\Gamma_{\mu} f=0, \quad \mu=1, \ldots, m_{1} .
$$

The energy function $E_{L}(q \dot{q})=\dot{q}^{i} \mathscr{P}_{i}(q \dot{q})-L(q \dot{q})$ verifies condition (2.9). Therefore there exits a function $H_{c} \in \Lambda^{0}\left(T^{*} Q\right)$ such that

$$
\mathrm{FL}^{*} H_{c}=E_{L} \text {, }
$$

where $H_{c}$ is only unambiguously defined in $M_{0}$.

Let us proceed to the inversion of the Legendre transformation FL. Given a point $\left(q_{0}, \dot{q}_{0}\right) \in \mathrm{TQ}$ and its image under $\mathrm{FL}\left(q_{0}, p_{0}\right), p_{0}=\left(q_{0}, \dot{q}_{0}\right)$, we have the identity

$$
H_{c}\left(q_{0}, \mathscr{P}\left(q_{0} \dot{q}_{0}\right)\right) \equiv q_{0}^{i} \mathscr{P}_{i}\left(q_{0}, \dot{q}_{0}\right)-L\left(q_{0}, \dot{q}_{0}\right),
$$

from which, taking the derivative with respect to $\dot{q}_{j}$, we obtain

$$
\frac{\partial H_{c}}{\partial p_{i}}\left(q_{0} p_{0}\right) \frac{\partial \mathscr{P}_{i}}{\partial \dot{q}_{j}}\left(q_{0} \dot{q}_{0}\right)=\frac{\partial \mathscr{P}_{i}}{\partial \dot{q}_{j}}\left(q_{0} \dot{q}_{0}\right) \dot{q}_{0}^{i} .
$$

Therefore, $\dot{q}_{0}^{i}-\left(\partial H_{c} / \partial p\right)\left(q_{0} p_{0}\right)$ is a null vector of $W$ and can be written in terms of (2.7):

$$
\dot{q}_{0}^{i}-\frac{\partial H_{c}}{\partial p_{i}}\left(q_{0}, p_{0}\right)=v_{\mu}^{0} \gamma_{\mu}^{i}\left(q_{0} \dot{q}_{0}\right),
$$

for some parameters $v_{\mu}^{0}$. Note that $\left(q_{0} \dot{q}_{0}\right)$ is a particular point of anti-image $\mathrm{FL}^{-1}\left(q_{0}, p_{0}\right)$. The whole anti-image is a leaf of foliation, defined in $T Q$ by the equivalence relation

$$
x \sim x^{1} \leftrightarrow \mathrm{FL} x=\mathrm{FLx} x^{1} ; \quad x, x^{1} \in T Q .
$$

Consequently, $\mathrm{Ker} \mathrm{FL}_{*}$ at every point $x \in T Q$ is given by the elements of $T_{x}(T Q)$ tangent to the leaves of the foliation previously defined. In other words, these leaves are the integral surfaces of the vector fields belonging to $\mathrm{Ker} F L_{*}$. This means that $\mathrm{FL}^{-1}\left(q_{0} p_{0}\right)$ will be generated from the point $\left(q_{0}, \dot{q}_{0}\right)$ by the exponential map $e^{u_{\mu} \Gamma_{\mu}}$ with $u_{\mu}$ arbitrary parameters and $\Gamma_{\mu}$ the vector fields (2.8). Therefore,

$$
\mathrm{FL}^{-1}\left(q_{0} p_{0}\right)=\left(q_{0}, \dot{q}(v)\right),
$$

where

$$
\dot{q}^{i}(v)=\frac{\partial H_{c}}{\partial p_{i}}\left(q_{0}, p_{0}\right)+v_{\mu} \frac{\partial \Phi_{\mu}^{(0)}}{\partial p_{i}}\left(q_{0} p_{0}\right),
$$

with the arbitrary parameters $v_{\mu}$ given by $v_{\mu}=v_{\mu}^{0}+u_{\mu}$. Due to the condition (2.4) given a point of this leaf we can determine the parameters $v^{\mu}$ in terms of the coordinates of this point. This means that for a given point $(q, p) \in M_{0}$ and all its possible anti-images we have the relation

$$
\dot{q}_{i}=\frac{\partial H_{c}}{\partial p_{i}}(q, p)+v_{\mu}(q \dot{q}) \frac{\partial \Phi_{\mu}^{(0)}}{\partial p_{i}}(q, p) .
$$

If we now consider Eq. (2.17) as a system with $(q, p)$ as data and $\dot{q}$ as unknowns, we show in Appendix A that there are no solutions if the data are out of $M_{0}$; whereas if the ( $q p) \in M_{0}$, the solutions of (2.17) are obviously given by (2.16). Therefore we conclude that the relations (2.17) and (2.2) are equivalent. Therefore Eq. (2.17) is the inverse Legendre transformation; note that Eq. (2.17) is an implicit equation for $\dot{q}$.

Let us observe that the application of $\Gamma$ to both sides of (2.17) gives

$$
\Gamma_{\mu} v_{v}=\delta_{\mu v} \text {. }
$$

This means that all the functions $v_{\mu}(q \dot{q})$ are not FL-projectable. However, we shall see in Sec. III that some of these 
functions admit a canonical form when restricted to a suitable submanifold of $T Q$.

Now if we take the derivative of (2.11) with respect to $q_{i}$, we have

$-\frac{\partial L}{\partial q^{i}}(q \dot{q})=\frac{\partial H_{c}}{\partial q^{i}}(q p)-\left(\dot{q}_{j}-\frac{\partial H_{c}}{\partial p_{j}}(q p)\right) \frac{\partial \mathscr{P}_{j}}{\partial q}(q, \dot{q})$,

where $(q p)=\mathrm{FL}(q \dot{q})$. If we use Eq. (2.17) we have

$-\frac{\partial L}{\partial q^{i}}(q \dot{q})=\frac{\partial H_{c}}{\partial q^{i}}(q p)-v_{\mu}(q \dot{q}) \frac{\partial \Phi_{\mu}^{(0)}}{\partial p_{j}}(q p) \frac{\partial \mathscr{P}_{j}}{\partial q^{i}}(q \dot{q})$,

but since $F L * \Phi_{\mu}=0$ we have

$$
\mathrm{FL}^{*}\left(\frac{\partial \Phi_{\mu}}{\partial p_{j}}\right) \frac{\partial \mathscr{P}_{j}}{\partial q^{i}}+\mathrm{FL}^{*}\left(\frac{\partial \Phi_{\mu}}{\partial q^{i}}\right)=0
$$

Therefore, Eq. (2.20) can be written as

$$
-\frac{\partial L}{\partial q^{i}}(q \dot{q})=\frac{\partial H_{c}}{\partial q^{i}}(q, p)+v_{\mu}(q \dot{q}) \frac{\partial \Phi_{\mu}^{(0)}}{\partial q^{i}}(q, p) \text {. }
$$

Let us now consider the equations of motion.

\section{B. Equations of motion}

A curve $q: I \in R \rightarrow Q$ is a solution of the Euler-Lagrange equations (2.1) if the function $p(t)$ defined by

$$
p(t)=\mathscr{P}\left(q(t), \frac{d q(t)}{d t}\right)
$$

satisfies

$$
\frac{d p}{d t}=\frac{\partial L}{\partial q}\left(q(t), \frac{d q}{d t}\right)
$$

Due to the equivalence between Eqs. (2.2) and (2.17) we can write an expression equivalent to (2.23), i.e.,

$\frac{d q}{d t}=\frac{\partial H_{c}}{\partial p}(q(t), p(t))+v_{\mu}\left(q(t), \frac{d q(t)}{d t}\right) \frac{\partial \Phi_{\mu}^{(0)}}{\partial p}(q(t), p(t))$,

also due to (2.22) we have

$$
\begin{aligned}
-\frac{\partial L}{\partial q}(q(t), \dot{q}(t))= & \frac{\partial H_{c}}{\partial q}(q(t), p(t))+v_{\mu}\left(q(t), \frac{d q(t)}{d t}\right) \\
& \times \frac{\partial \Phi_{\mu}^{(0)}}{\partial q}(q(t), p(t)),
\end{aligned}
$$

and using (2.24), Eq. (2.26) is written as

$$
\begin{aligned}
-\frac{d p}{d t}= & \frac{\partial H_{c}}{\partial q}(q(t), p(t)) \\
& +v_{\mu}\left(q(t), \frac{d q(t)}{d t}\right) \frac{\partial \Phi_{\mu}^{(0)}}{\partial q}(q(t), p(t)) .
\end{aligned}
$$

Equations (2.25) and (2.27) are the Hamilton-Dirac equations for the singular Lagrangian $L(q \dot{q})$, so we can formulate the following theorem.

Theorem: If $q(t)$ is a solution of Euler-Lagrange equations (2.1) in configuration space, the lifting to $T^{*} Q$ given by $(q(t), p(t))$ with $p(t)$ defined by (2.2), is a solution of the Hamilton-Dirac equations (2.25) and (2.27).

Furthermore, in the inverse sense, if $(q(t), p(t))$ verify
Eq. (2.25) and Eq. (2.27), then (2.23) is satisfied because Eq. (2.23) is equivalent to (2.25). Furthermore, from (2.26) and (2.27) we obtain (2.24), therefore the following theorem holds.

Theorem 2: If $(q(t), p(t))$ is a solution of the HamiltonDirac equations (2.25) and (2.27), then $q(t)$ verifies the Euler-Lagrange equations (2.1).

If we consider the canonical symplectic structure of $T^{*} Q$ we can write Eqs. (2.25) and (2.27) in terms of PB as

$$
\begin{aligned}
& \frac{d q}{d t}=\left\{q, H_{c}\right\}+v_{\mu}\left(q, \frac{d q}{d t}\right)\left\{q, \Phi_{\mu}^{(0)}\right\}, \\
& \frac{d p}{d t}=\left\{p, H_{c}\right\}+v_{\mu}\left(q, \frac{d q}{d t}\right)\left\{p, \Phi_{\mu}^{(0)}\right\} .
\end{aligned}
$$

These equations are not written in the normal form, in the same sense as the Euler-Lagrange equations of motion, (2.1), therefore the possible solutions of those equations lie in a submanifold of $T^{*} Q$ and the solution passing through a point of that submanifold is not necessarily unique.

Equations (2.28) and (2.39) can be written in a normal form if one introduces $m_{1}$ arbitrary functions of the evolution parameter $\lambda_{\mu}(t)$, and also imposes from the outset the primary constraints

$$
\begin{aligned}
& \frac{d q}{d t} \underset{M_{0}}{=}\left\{q, H_{c}\right\}+\lambda_{\mu}(t)\left\{q, \Phi_{\mu}^{(0)}\right\}, \\
& \frac{d p}{d t} \underset{M_{0}}{=}\left\{p, H_{c}\right\}+\lambda_{\mu}(t)\left\{p, \Phi_{\mu}^{(0)}\right\}
\end{aligned}
$$

where $=$ means weak equality on the surface $M_{0}$. Equations (2.31) are the standard Hamilton-Dirac equations. ${ }^{1,2,4,5}$

\section{HAMILTONIAN FORMALISM}

In the preceding section we assumed the existence of solutions of the equations of motion and we have shown the equivalence between the Lagrangian and Hamiltonian formalism. Now we study the submanifold where those solutions exist, we will use an iterative procedure. Let us begin with the Hamiltonian formalism, the Hamilton-Dirac equations of motion are Eqs. (2.28) and (2.29):

$$
\begin{aligned}
& \frac{d q}{d t}=\left\{q, H_{c}\right\}+v_{\mu}\left(q, \frac{d q}{d t}\right)\left\{q, \Phi_{\mu}^{(0)}\right\}, \\
& \frac{d p}{d t}=\left\{p, H_{c}\right\}+v_{\mu}\left(q, \frac{d q}{d t}\right)\left\{p, \Phi_{\mu}^{(0)}\right\},
\end{aligned}
$$

where $\Phi_{\mu}^{(0)}$ are the primary Hamiltonian constraints and $v_{\mu}(q, \dot{q})$ are known function of $q$ and $\dot{q}$. We know, from Appendix $A$, that (3.1) have only solutions if the initial conditions belong to the submanifold $M_{0} \subset T^{*} Q$. In that case, a curve passing through a point of $M_{0}$ will be a solution of (3.1) if

$$
\frac{d \Phi_{\mu}^{(0)}}{d t} \underset{M_{0}}{=} 0, \quad \mu=1, \ldots, m_{1},
$$

that is, the solution $(q(t), p(t))$ must belong entirely to $M_{0}$. In general, Eqs. (3.2) will be restrictions for the initial conditions. We write (3.2) as

$$
0 \underset{M_{0}}{=}\left\{\Phi_{\mu}^{(0)}, H_{c}\right\}+v_{\nu}(q, \dot{q})\left\{\Phi_{\mu}^{(0)} \Phi_{v}^{(0)}\right\}
$$


To discuss the content of (3.2) it is necessary to know the rank of the PB matrix between the primary constraints, i.e., $\operatorname{rank}\left|\left\{\Phi_{\mu}^{(0)}, \Phi_{\nu}^{(0)}\right\}\right|=m_{1}-m_{2}, \quad \mu, v=1, \ldots, m_{1}$,

which we assume to be constant on $\boldsymbol{M}_{0}$. It is convenient to introduce an equivalent set of constraints

$$
\begin{aligned}
& \Phi_{\mu_{0}}^{(0)}, \quad \mu_{0}=1, \ldots, m_{2}, \\
& \Phi_{\mu_{0}^{\prime}}^{(0)}, \quad \mu_{0}^{\prime}=1, \ldots, m_{1}-m_{2},
\end{aligned}
$$

with the properties

$$
\begin{aligned}
& 0 \neq \operatorname{det}\left|\left\{\Phi_{\mu_{0}^{\prime}}^{(0)}, \Phi_{\nu_{0}^{\prime}}^{(0)}\right\}\right| \equiv \operatorname{det} C_{\mu_{0}^{\prime} \nu_{0}^{\prime}}^{(1)}, \\
& \left\{\Phi_{\mu_{0}^{\prime}}^{(0)}, \Phi_{\mu_{0}}^{(0)}\right\} \underset{M_{0}}{=0,}\left\{\Phi_{\mu_{0}}^{(0)}, \Phi_{\nu_{0}}^{(0)}\right\}_{M_{0}}^{=} 0,
\end{aligned}
$$

therefore $\Phi_{\mu_{0}}^{(0)}, \Phi_{\mu_{0}^{\prime}}^{(0)}$ are first and second class, respectively, on $M_{0}$. Note that $\Phi_{\mu_{0}^{\prime}}^{(0)}$ are $m_{1}-m_{2}$ of the old primary constraints $\Phi_{\mu}^{(0)}$, instead $\Phi_{\mu_{0}}^{(0)}$ are linear combinations of them. If we consider Eq. (3.2) for the second-class constraints $\Phi_{\mu_{0}^{\prime}}^{(0)}$, we obtain a canonical expression for the functions $v_{v_{0}}(q, \dot{q})$ :

$$
v_{v_{0}^{\prime}}(q \dot{q}) \underset{M_{0}}{=}-\left(C^{(1)}\right)_{v_{0}^{\prime} \mu_{0}^{\prime}}^{-1}\left\{\Phi_{\mu_{0}^{\prime}}, H_{c}\right\} .
$$

Therefore the evolution for a generic quantity $A(q, p)$ in $M_{0}$ is given by

$\frac{d A}{d t} \underset{M_{0}}{=}\left\{A, H_{c}^{(1)}\right\}+v_{v_{0}}(q \dot{q})\left\{A, \Phi_{v_{0}}^{(0)}\right\}, \quad v_{0}=1, \ldots, m_{2}$,

where

$$
\begin{gathered}
H_{c}^{(1)} \equiv H_{c}-\left\{H_{c} \Phi_{\mu_{0}^{\prime}}^{(0)}\right\}\left(C^{(1)}\right)_{\mu_{0}^{\prime} v_{0}^{\prime}}^{-1} \Phi_{v_{0}^{\prime}}^{(0)}, \\
\mu_{0}^{\prime}, v_{0}^{\prime}=1, \ldots, m_{1}-m_{2},
\end{gathered}
$$

with the properties ${ }^{13}$

$$
\left\{\Phi_{\mu_{0}^{\prime}}^{(0)}, H_{c}^{(1)}\right\} \underset{M_{0}}{=} 0 .
$$

The evolution of the first class constraints $\Phi_{\mu_{0}}^{(0)}$ is given by

$$
\frac{d}{d t} \Phi_{\mu_{0}}^{(0)} \underset{\bar{M}_{0}}{=}\left\{\Phi_{\mu_{0}}^{(0)}, H_{c}^{(1)}\right\} \equiv \Phi_{\mu_{0}}^{(1)},
$$

the stability conditions for $\Phi_{\mu_{0}}^{(1)}$ are

$$
\Phi_{\mu_{0}}^{(1)}=0 .
$$

If all these conditions are satisfied on $M_{0}$ the analysis is finished, if this is not the case Eqs. (3.13) are new restrictions on the initial conditions, which we call secondary constraints. Note that some of these constraints can be automatically satisfied on $M_{0}$, but in order to use a more compact notation we will continue to use the subscript $\mu_{0}$ for all secondary constraints.

Let $M_{1}$, be the new submanifold defined by

$$
\begin{array}{ll}
\Phi_{\mu_{0}^{\prime}}^{(0)}=0, & \mu_{0}^{\prime}=1, \ldots, m_{1}-m_{2}, \\
\Phi_{\mu_{0}}^{(0)}=0, & \Phi_{\mu_{0}}^{(1)}=0, \quad \mu_{0}=1, \ldots, m_{2} .
\end{array}
$$

A curve passing through a point of $M_{1}$ will be a solution of (3.1) if

$$
\frac{d \Phi_{\mu_{0}}^{(1)}}{d t}=0
$$

These stability conditions can be written explicitly

$$
0 \underset{M_{1}}{=}\left\{\Phi_{\mu_{0}}^{(1)}, H_{c}^{(1)}\right\}+v_{v_{0}(q, q)}\left\{\Phi_{\mu_{0}}^{(1)}, \Phi_{v_{0}}^{(0)}\right\}
$$

In Eq. (3.15) a PB matrix appears between the primary firstclass constraints on $\boldsymbol{M}_{0}$ and the secondary constraints. As is shown in Appendix B this matrix is symmetric:

$$
\left\{\Phi_{\mu_{0}}^{(1)}, \Phi_{v_{0}}^{(0)}\right\} \underset{M_{1}}{=}\left\{\Phi_{v_{0}}^{(1)}, \Phi_{\mu_{0}}^{(0)}\right\} .
$$

Let $m_{2}-m_{3}$ be the rank of this matrix. Due to this symmetry property we can introduce a new set of constraints

$$
\begin{aligned}
& \Phi_{\mu_{1}}^{(0)}=0, \quad \Phi_{\mu_{1}}^{(1)}=0, \quad \mu_{1}=1, \ldots, m_{3}, \\
& \Phi_{\mu_{1}^{\prime}}^{(0)}=0, \quad \Phi_{\mu_{1}^{\prime}}^{(1)}=0, \quad \mu_{1}^{\prime}=1, \ldots, m_{2}-m_{3},
\end{aligned}
$$

with the following properties:

$$
\begin{aligned}
& 0 \neq \underset{M_{1}}{\operatorname{det}}\left|\left\{\Phi_{\mu_{1}^{\prime}}^{(1)}, \Phi_{\nu_{1}^{\prime}}^{(0)}\right\}\right| \equiv \operatorname{det} C_{\mu_{1}^{\prime} v_{i}^{\prime}}^{(2)}, \\
& \left\{\Phi_{\mu_{1}}^{(1)}, \Phi_{\mu_{0}}^{(0)}\right\} \underset{M_{1}}{=} 0, \\
& \left\{\Phi_{\mu_{0}}^{(1)}, \Phi_{\mu_{1}}^{(0)}\right\} \underset{M_{1}}{=} 0 .
\end{aligned}
$$

Note that $\Phi_{\mu_{1}^{\prime}}^{(0)}$ are $m_{2}-m_{3}$ of the old $\Phi_{\mu_{0}}^{(0)}$, due to the symmetry property, Eq. (3.16), and $\Phi_{\mu_{1}^{\prime}}^{(1)}$ are also the same $m_{2}-m_{3}$ of the $\Phi_{\mu_{0}}^{(1)}$. The $\Phi_{\mu_{1}}^{(0)}$ are a linear combination of the $\Phi_{\mu_{0}}^{(0)}$ and the $\Phi_{\mu_{1}}^{(1)}$ are the same linear combination of the $\Phi_{\mu_{0}}^{(1)}$. This means that we also have

$$
\begin{aligned}
& \Phi_{\mu_{1}}^{(1)}=\left\{\Phi_{\mu_{1}}^{(0)}, H_{c}^{(1)}\right\}, \\
& \Phi_{\mu_{1}^{(1)}}^{(1)}=\left\{\Phi_{\mu_{1}^{\prime}}^{(0)}, H_{c}^{(1)}\right\},
\end{aligned}
$$

This means that the labeling of new constraints is compatible with their stability, therefore we have a sort of hereditary property. It should be noted that $\Phi_{\mu_{1}}^{(0)}$ are first-class constraints on $M_{1}$.

At this point, we consider the stability condition for the constraints $\Phi_{v_{1}^{\prime}}^{(1)}$. From this we obtain a canonical expression for the functions $v_{v_{1}^{\prime}}(q \dot{q})$ :

$$
v_{v_{i}^{\prime}}(q \dot{q}) \underset{M_{1}}{=}-\left(C^{(2)}\right)_{v_{i}^{\prime} \mu_{i}^{\prime}}^{-1}\left\{\Phi_{\mu_{i}^{\prime}}^{(1)}, H_{c}^{(1)}\right\} .
$$

The evolution on $M_{1}$ is given by

$$
\frac{d A}{d t} \underset{M_{1}}{=}\left\{A, H_{c}^{(2)}\right\}+v_{v_{1}}(q \dot{q})\left\{A, \Phi_{v_{1}}^{(0)}\right\},
$$

where

$$
H_{c}^{(2)}=H_{c}^{(1)}+\left\{H_{c}^{(1)}, \Phi_{\mu_{1}^{\prime}}^{(1)}\right\}\left(C^{(2)}\right)_{\mu_{1}^{\prime} v_{1}^{\prime}}^{-1} \Phi_{v_{1}^{\prime}}^{(0)},
$$

with the properties

$$
\left\{\Phi_{\mu_{1}^{\prime}}^{(1)}, H_{c}^{(2)}\right\} \underset{M_{1}}{=} 0 .
$$

Now consider the stability of the remaining secondary constraints

$$
\frac{d}{d t} \Phi_{\mu_{1}}^{(1)} \underset{M_{1}}{=}\left\{\Phi_{\mu_{1}}^{(1)}, H_{c}^{(2)}\right\} \equiv \Phi_{\mu_{1}}^{(2)},
$$

the relations $\Phi_{\mu_{1}}^{(2)}=0$ can be satisfied on $M_{1}$ in which case the analysis is finished. Otherwise

$$
\Phi_{\mu_{1}}^{(2)}=0, \quad \mu_{1}=1, \ldots, m_{3},
$$

are tertiary constraints. At this level the evolution is restricted to the submanifold $M_{2}$ : 


$$
\begin{array}{lll}
\Phi_{\mu_{0}^{\prime}}^{(0)}=0, & \Phi_{\mu_{1}^{\prime}}^{(0)}=0, & \Phi_{\mu_{1}}^{(0)}=0, \\
\Phi_{\mu_{i}^{\prime}}^{(1)}=0, & \Phi_{\mu_{1}}^{(0)}=0, & \Phi_{\mu_{1}}^{(2)}=0,
\end{array}
$$

with

$\mu_{0}^{\prime}=1, \ldots, m_{1}-m_{2}, \quad \mu_{1}^{\prime}=1, \ldots, m_{2}-m_{3}, \quad \mu_{1}=1, \ldots, m_{3}$.

In order to study the stability of tertiary constraints $\Phi_{\mu}^{(2)}$, we need to consider the PB matrix of the primary firstclass constraints on $M_{1}, \Phi_{v_{1}}^{(0)}$, with the tertiary constraints $\Phi_{\mu_{1}}^{(2)}$. As is shown in Appendix B, this matrix is antisymmetric in the submanifold $M_{2}$ :

$$
\left\{\Phi_{\mu_{1}}^{(2)}, \Phi_{v_{1}}^{(0)}\right\} \underset{M_{2}}{=}-\left\{\Phi_{v_{1}}^{(2)}, \Phi_{\mu_{1}}^{(0)}\right\}
$$

Let $m_{3}-m_{4}$ be the rank of that matrix. Due to the antisymmetry property we can introduce a set of constraints

$$
\begin{aligned}
& \Phi_{\mu_{2}}^{(2)}, \Phi_{\mu_{2}^{\prime}}^{(2)}, \quad \mu_{2}=1, \ldots, m_{4}, \\
& \Phi_{\mu_{2}}^{(0)}, \Phi_{\mu_{2}^{\prime}}^{(0)}, \quad \mu_{2}^{\prime}=1, \ldots, m_{3}-m_{4},
\end{aligned}
$$

which define the same surface as the set $\Phi_{\mu_{1}}^{(0)}, \Phi_{v_{1}}^{(2)}$, with the properties

$$
\begin{aligned}
& \operatorname{det} C_{\mu_{2}^{\prime} \nu_{2}^{\prime}}^{(3)} \equiv \operatorname{det}\left\{\Phi_{\mu_{2}^{\prime}}^{(2)}, \Phi_{\nu_{2}^{\prime}}^{(0)}\right\}_{M_{2}}^{\neq 0}, \\
& \left\{\Phi_{\mu_{2}}^{(2)} \Phi_{\mu_{1}}^{(0)}\right\}=0, \quad\left\{\Phi_{\mu_{1}}^{(2)}, \Phi_{\mu_{2}}^{(0)}\right\}=\underset{M_{2}}{=0}, \\
& \Phi_{\mu_{2}}^{(1)}=\left\{\Phi_{\mu_{2}}^{(0)}, H_{c}^{(1)}\right\}, \quad \Phi_{\mu_{2}^{\prime}}^{(1)}=\left\{\Phi_{\mu_{2}^{\prime}}^{(0)}, H_{c}^{(1)}\right\}, \\
& \Phi_{\mu_{2}}^{(2)}=\left\{\Phi_{\mu_{2}}^{(1)} H_{c}^{(2)}\right\}, \quad \Phi_{\mu_{2}^{\prime}}^{(2)}=\left\{\Phi_{\mu_{2}^{\prime}}^{(1)}, H_{c}^{(2)}\right\} .
\end{aligned}
$$

The stability conditions for the tertiary constraints $\Phi_{\mu_{2}^{\prime}}^{(2)}$, as in the previous case, enables us to obtain a canonical expression for the functions $v_{v_{2}{ }^{.}}$. Using that expression the evolution on $M_{2}$ is given by

$$
\frac{d A}{d t} \underset{M_{2}}{=}\left\{A, H_{c}^{(3)}\right\}+v_{v 2}\left\{A, \Phi_{v_{2}}^{(0)}\right\}
$$

where

$$
H_{c}^{(3)}=H_{c}^{(2)}-\left\{H_{c}^{(2)}, \Phi_{\mu_{2}^{\prime}}^{(2)}\right\}\left(C^{(3)}\right)_{\mu_{2}^{\prime} \nu_{2}^{\prime}}^{-1} \Phi_{\nu_{2}^{\prime}}^{(0)},
$$

with the property

$$
\left\{\Phi_{\mu_{2}^{\prime}}^{(2)}, H_{c}^{(3)}\right\} \underset{M_{2}}{=} 0
$$

With respect to the stability of $\Phi_{\mu_{2}}^{(2)}$ we have the relations

$$
\Phi_{\mu_{2}}^{(3)}=0,
$$

where

$$
\Phi_{\mu_{2}}^{(3)} \equiv\left\{\Phi_{\mu_{2}}^{(2)}, H_{c}^{(3)}\right\}
$$

If the relations (3.39) are verified on $M_{2}$, the analysis is finished. Otherwise we have more constraints and therefore we need further to require the stability of those constraints and the procedure continues as before. Let assume that our Lagrangian has a final submanifold $M_{f}$ where we have solution of the equations of motion (3.1). We write the constraints defining $M_{f}$ as

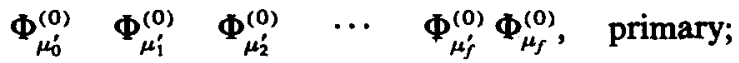

$$
\begin{aligned}
& \Phi_{\mu_{1}^{\prime}}^{(1)} \quad \Phi_{\mu_{2}^{\prime}}^{(1)} \quad \cdots \quad \Phi_{\mu_{f}^{\prime}}^{(1)} \Phi_{\mu_{f}}^{(1)}, \quad \text { secondary; } \\
& \boldsymbol{\Phi}_{\mu_{i}^{\prime}}^{(2)} \quad \cdots \quad \Phi_{\mu_{j}^{\prime}}^{(2)} \Phi_{\mu_{f}}^{(2)}, \quad \text { tertiary; } \\
& \Phi_{\mu_{f}^{\prime}}^{(0)} \Phi_{\mu_{f}}^{(n)}, f \text {-ary, }
\end{aligned}
$$

where the hereditary property is manifest. The equations of motion on $M_{f}$ are

$$
\frac{d A}{d t} \underset{M_{f}}{=}\left\{A, H_{c}^{(f+1)}\right\}+v_{v_{f}}(q \dot{q})\left\{A \Phi_{v_{f}}^{(f)}\right\},
$$

where

$H^{(f+1)}=H_{c}^{(n)}+(-)^{(f+1)}\left\{H_{c}^{(n)}, \Phi_{\mu_{f}^{\prime}}^{(n)}\right\}\left(C^{(n)}\right)_{\mu_{f}^{\prime} v_{f}^{\prime}}^{-1} \Phi_{v_{f}^{\prime}}^{(0)}$.

The matrix $C^{(f)}$ is symmetric or antisymmetric depending on whether $f$ is odd or even.

We have

$$
\left\{\Phi_{\mu_{f}^{\prime}}^{(f)}, H_{c}^{(f+1)}\right\} \underset{M_{f}}{=} 0
$$

and since the analysis is finished we have

$$
\left\{\Phi_{\mu_{f}}^{(n)}, H_{c}^{(f+1)}\right\} \underset{M_{f}}{=} 0
$$

Note that in the equations of motion there appear functions $v_{\mu_{f}}(q \dot{q})$ that are not determined canonically and are associated with the primary first class constraints on the submanifold $M_{f}$.

Let us now study the relation between the procedure of Dirac brackets (DB) for second class constraints, ${ }^{1}$ and the procedure developed here. Let us begin with the case with no tertiary constraints. The DB with respect to second-class constraints $\Phi_{\mu_{0}^{\prime}}^{(0)} \Phi_{\mu_{1}^{\prime}}^{(0)} \Phi_{\mu_{i}^{\prime}}^{(1)}$ can be constructed in two steps. First we construct the DB for the constraints $\Phi_{\mu_{0}^{\prime}}^{(0)}$, i.e.,

$$
\{A, B\}^{H_{1}} \equiv\{A, B\}-\left\{A \Phi_{\mu_{0}^{\prime}}^{(0)}\right\}\left(D^{(1)}\right)_{\mu_{0}^{\prime} v_{0}^{\prime}}^{-1}\left\{\Phi_{v_{0}^{\prime}}^{(0)}, B\right\},
$$

where $\left(\Delta^{(1)}\right)^{-1}$ is the inverse matrix of $D^{(1)}$ defined by

$$
D_{\mu_{0}^{\prime} v_{0}^{\prime}}^{(1)}=\left\{\Phi_{\mu_{0}^{\prime}}^{(0)}, \Phi_{v_{0}^{\prime}}^{(0)}\right\}
$$

which coincides with $C_{\mu_{0}^{\prime} \nu_{0}^{\prime}}^{(1)}$ [Eq. (3.6)]. The final DB is written as

$$
\{A, B\}^{H_{2}} \equiv\{A, B\}^{H_{1}}-\left\{A \chi_{\mu_{1}^{\prime}}\right\}^{H_{1}} D_{\mu_{1}^{\prime} v_{1}^{\prime}}^{(2)}\left\{\chi_{v_{1}^{\prime}}, B\right\}^{H_{1}} \text {, }
$$

where $\chi_{\mu_{1}^{\prime}}$ indicates any one of the constraints $\Phi_{\mu_{1}^{\prime}}^{(0)}, \Phi_{\mu_{1}^{\prime}}^{(1)}$, and $\left(D^{(2)}\right)^{-1}$ is the inverse of the matrix $D^{(2)}$ defined by $D_{\mu_{i}^{\prime} v_{i}^{\prime}}^{(2)}=\left\{\chi_{\mu_{i}^{\prime}}, \chi_{v_{i}^{\prime}}\right\}^{H_{1}}$. Explicitly

$$
D^{(2)}=\left(\begin{array}{cc}
0 & -C^{(2)} \\
C^{(2)} & K
\end{array}\right),
$$

where $C^{(2)}$ is the matrix defined in (3.18) and $K$ is a matrix constructed with the $\Phi_{\mu_{i}^{\prime}}^{(1)}$ constraints.

Let us consider (3.47) in the case $B=H_{c}$. Using Eqs. (3.48), (3.12), and (3.24) we have 


$$
\begin{aligned}
\left\{A, H_{c}\right\}^{H_{2}} \underset{M_{0}}{=} & \left\{A, H_{c}\right\}^{H_{1}}-\left\{A \Phi_{\mu_{1}^{\prime}}^{(0)}\right\}^{H_{c}}\left[\left(C^{(2)}\right) K\left(C^{(2)}\right)^{-1}\right]_{\mu_{1}^{\prime} v_{1}^{\prime}}\left\{\Phi_{\nu_{1}^{\prime}}^{(0)} H_{c}\right\}^{H_{1}} \\
& -\left\{A \Phi_{\mu_{1}^{(0)}}^{(0)} H^{H_{1}}\left(C^{(2)}\right)_{\mu_{1}^{\prime} v_{1}^{\prime}}^{-1}\left\{\Phi_{\nu_{1}^{\prime}}^{(1)}, H_{c}\right\}^{H_{1}}+\left\{A \Phi_{\mu_{1}^{\prime}}^{(1)}\right\}^{H_{1}}\left(C^{(2)}\right)_{\mu_{1}^{\prime} v_{1}^{\prime}}^{-1}\left\{\Phi_{\mu_{1}^{\prime}}^{(0)}, H_{c}\right\}^{H_{1}} \underset{M_{1}}{=}\left\{A, H_{c}^{(2)}\right\} .\right.
\end{aligned}
$$

Therefore our procedure on the $M_{1}$ surface is equivalent to the Dirac procedure. In Appendix $C$ we explicitly prove this result for the case of no quartiary constraints. In order to give a proof in the general case, we need to consider a more geometrical formulation that takes into account the new structures we have found. Work in that direction is in progress.

Summing up, Eqs. (3.42) are equivalent on $M_{f}$ to the equations of motion generated by the total Dirac Hamiltonian:

$$
H_{T}=H^{H(f+1)}+v_{\mu_{f}} \Phi_{\mu_{f}}^{(0)},
$$

where $H^{H(f+1)}$ is the starred Hamiltonian ${ }^{13}$ with respect to all second-class constraints. Therefore the DB is not the minimal structure to obtain the Hamiltonian equations of motion.

\section{LAGRANGIAN FORMALISM: RELATION BETWEEN THE LAGRANGIAN AND HAMILTONIAN CONSTRAINTS}

In the previous section we have built a new scheme for the construction and classification of the submanifold of the Hamiltonian constraints. Now, we shall use these results to do the same with the Lagrangian constraints.

Using the Hessian matrix $W_{i j}$, we can write the Lagrangian equations of motion (2.1) as

$$
W_{i j} \ddot{q}^{j}=\alpha_{i},
$$

where

$$
\alpha_{i} \equiv \frac{\partial L}{\partial q^{i}}-\dot{q}^{j} \frac{\partial^{2} L}{\partial q^{j} \partial \dot{q}^{i}} .
$$

If the rank of $W$ is $n-m_{1}, m_{1}>0$, the Hessian will have $m_{1}$ null vectors $\gamma_{\mu}(q, \dot{q})$ such that

$$
W_{i j} \gamma_{\mu}^{j}=0 \text {. }
$$

The contraction of Eq. (4.1) with a null vector gives

$$
\chi_{\mu}^{(1)} \equiv \alpha_{i} \gamma_{\mu}^{i}=0 \text {. }
$$

This is the first generation of Lagrangian constraints:

$$
\chi_{\mu}^{(1)}=0, \quad \mu=1, \ldots, m_{1} .
$$

The submanifold in $T Q$ locally defined by the vanishing of the $\chi_{\mu}^{(1)}$ is denoted by $S_{1}$. These Lagrangian constraints can also be obtained with the help of the operator $K$ :

$$
K=\dot{q}^{i} \mathrm{FL}^{*} \frac{\partial}{\partial q^{i}}+\frac{\partial L}{\partial q^{i}} \mathrm{FL}^{*} \frac{\partial}{\partial p_{i}},
$$

which takes a function in $\Lambda^{0}\left(T^{*} Q\right)$, differentiates it with respect to time, and gives the result in $\Lambda^{0}(T Q)$. As we demonstrate in Appendix $\mathrm{C}$, one has

$$
K \Phi_{\mu}^{(0)}=\chi_{\mu}^{(1)} \text {. }
$$

From this equation, we can see that every primary constraint produces a Lagrangian constraint of the first generation. Be- cause of the linearity of the relations (4.4) and (2.7), the classification (3.5) enables us to make the splitting

$$
\begin{array}{ll}
\chi_{\mu_{0}}^{(1)}, & \mu_{0}=1, \ldots, m_{2}, \\
\chi_{\mu_{0}^{\prime}}^{(1)}, & \mu_{0}^{\prime}=1, \ldots, m_{1}-m_{2} .
\end{array}
$$

Now we can demonstrate the following relations:

$$
\mathbf{F L} *\left\{\Phi_{\mu_{0}^{\prime}}^{(0)}, H_{c}\right\}+v_{v_{0}^{\prime}}(q, \dot{q}) F L^{*}\left\{\Phi_{\mu_{0}^{\prime}}^{(0)}, \Phi_{v_{0}^{\prime}}^{(0)}\right\}=\Phi_{\mu_{0}^{\prime}}^{(0)},
$$

$$
\mathrm{FL}^{*} \Phi_{\mu_{0}}^{(1)}=\chi_{\mu_{0}}^{(1)}\left(\bmod \chi_{\mu_{0}^{\prime}}^{(1)}\right)
$$

First, we demonstrate (4.9a), using (2.17), (2.20), (D4), and (D5). We have

$$
\begin{aligned}
\mathrm{FL}^{*}\left\{\Phi_{\mu_{0}^{\prime}}^{(0)}, H_{c}\right\}= & \chi_{\mu_{0}^{\prime}}-v_{v_{0}}(q, \dot{q}) \mathrm{FL} *\left\{\Phi_{\mu_{0}^{\prime}}^{(0)}, \Phi_{v_{0}}^{(0)}\right\} \\
& -v_{v_{0}^{\prime}}(q, \dot{q}) \mathrm{FL} *\left\{\Phi_{\mu_{0}^{\prime}}^{(0)}, \Phi_{v_{0}^{\prime}}^{(0)}\right\},
\end{aligned}
$$

but $F L *\left\{\Phi_{\mu_{0}^{\prime}}^{(0)}, \Phi_{\mu_{v_{0}}^{0}}^{(0)}\right\}=0$, due to the fact $\left\{\Phi_{\mu_{0}^{(0)}}^{(0)}, \Phi_{v_{0}}^{(0)}\right\}=0$, so (4.9a) is demonstrated. Now we can demonstrate (4.9b). We have $\Phi_{\mu_{0}}^{(1)}=\left\{\Phi_{\mu_{0}}^{(0)}, H_{c}^{(1)}\right\}$ with $H_{c}^{(1)}$ given by (3.12); using (2.17) and (2.20) we have

$\mathrm{FL}^{*} \frac{\partial H_{c}^{(1)}}{\partial p_{i}}=\dot{q}^{i}-v_{\mu_{0}}(q \dot{q}) \mathrm{FL}^{*} \frac{\partial \Phi \mu_{0}^{(0)}}{\partial p_{i}}\left(\bmod \Phi_{\mu_{0}^{\prime}}^{(1)}\right)$,

$\mathrm{FL}^{*} \frac{\partial H_{c}^{(1)}}{\partial q^{i}}$

$$
=-\frac{\partial L}{\partial q^{i}}-v_{\mu_{0}}(q, \dot{q}) \mathrm{FL}^{*} \frac{\partial \Phi_{\mu_{0}}^{(0)}}{\partial q_{i}}\left(\bmod \chi_{\mu_{0}^{\prime}}^{(1)}\right),
$$

and therefore

$$
\begin{aligned}
\mathrm{FL}^{*} & \left\{\Phi_{\mu_{0}}^{(0)}, H_{c}^{(1)}\right\} \\
& =\chi_{\mu_{0}}^{(1)}-v_{\nu_{\mathrm{o}}}(q, \dot{q}) \mathrm{FL} *\left\{\Phi_{\mu_{0}}^{(0)}, \Phi_{\nu_{0}}^{(0)}\right\} \quad\left(\bmod \chi_{\mu_{a}^{\prime}}^{(1)}\right) \\
& =\Phi_{\mu_{0}}^{(1)}\left(\bmod \chi_{\mu_{0}^{\prime}}^{(1)}\right)
\end{aligned}
$$

as desired.

Furthermore from an analogous equation to (4.10) we have that

$$
\mathrm{FL} *\left\{\Phi_{\mu_{0}}^{(0)}, H_{c}\right\}=\chi_{\mu_{0}}^{(1)} .
$$

From Eq. (4.9a) we see that the constraints $\chi_{\mu_{0}^{\prime}}^{(1)}$ are not FLprojectable, instead the constraints $\chi_{\mu_{0}}^{(1)}$ are FL-projectable [see Eq. (4.12)]. These results can also be seen using the relation

$$
\Gamma_{\mu} \chi_{\nu}^{(1)}=\mathrm{FL} *\left\{\Phi_{\nu}^{(0)}, \Phi_{\mu}^{(0)}\right\}
$$

[see Appendix C and Eq. (2.9)]. Therefore the Lagrangian constraints $\chi_{\mu_{0}^{\prime}}^{(1)}$ associated to the primary second-class constraints by means of the operator $K$ are not FL-projectable. Instead those associated the primary first-class constraints are FL-projectable.

Now we want to investigate the stability of the constraints that locally define $S_{1}$. We have 


$$
\frac{d}{d t} \chi_{\mu}^{(1)}=\dot{q}^{i} \frac{\partial \chi_{\mu}^{(1)}}{\partial q^{i}}+\ddot{q}^{i} \frac{\partial \chi_{\mu}^{(1)}}{\partial \dot{q}^{i}}
$$

We can obtain $\ddot{q}$ from the equation of motion introducing the completeness relation ( $\operatorname{see} \mathrm{A} 5$ )

$$
\delta_{j}^{i}=M^{i k} W_{k j}+\tilde{\gamma}_{\mu j} \gamma_{\mu}^{i},
$$

we have

$$
\ddot{q}^{i} \underset{s_{1}}{=} M^{i j} \alpha_{j}+\beta_{\mu} \gamma_{\mu}^{i},
$$

where

$$
\beta_{\mu}=\tilde{\gamma}_{\mu k} \ddot{q}_{k},
$$

are the accelerations that are undetermined by the equations of motion. Substitution of (4.16) in (4.14) gives

$$
\frac{d}{d t} \chi_{\mu}^{(1)}{ }_{s_{1}} D^{(0)} \chi_{\mu}^{(1)}+\beta_{\nu} \Gamma_{\nu} \chi_{\mu}^{(1)},
$$

where

$$
D^{(0)} \equiv \alpha_{j} M^{j i} \frac{\partial}{\partial \dot{q}^{i}}+\dot{q}^{i} \frac{\partial}{\partial q^{i}} .
$$

If we consider the stability, Eq. (4.14), of the non-FL-projectable constraints, $\chi_{\mu_{0}^{\prime}}^{(0)}$, we have

$$
\underset{S_{1}}{=} D^{(0)} \chi_{\mu_{0}^{\prime}}^{(1)}+\beta_{v_{0}^{\prime}} \Gamma_{v_{0}^{0}} \chi_{\mu_{0}^{(1)}}^{(1)}
$$

with

$$
\Gamma_{v_{0}^{\prime}} \chi_{\mu_{0}^{\prime}}^{(1)}=\mathrm{FL}^{*}\left(C^{(1)}\right)_{\mu_{0}^{\prime} v_{0}^{\prime}},
$$

due to (4.13) and (3.6). Since $C^{(1)}$ has an inverse one can determine the undetermined accelerations $\beta_{v_{0}^{\prime}}$ as a function of $q$ and $\dot{q}$ :

$$
\beta_{\nu_{0}^{\prime}}=-\mathrm{FL}^{*}\left[\left(C^{1}\right)_{v \mu_{0}^{\prime}}^{-1}\right]^{D^{(0)}} \chi_{\mu_{0}^{(1)}}^{(1)}
$$

Let us now consider the stability of the FL-projectable constraints. We have

$$
\frac{d}{d t} \chi_{\mu_{0}}^{(1)} \bar{s}_{s_{1}} D^{(0)} \chi_{\mu_{0}^{\prime}}^{(1)} \equiv \chi_{\mu_{0}}^{(2)} \text {. }
$$

If the relations $\chi_{\mu_{0}}^{(2)}=0$ are automatically verified in $S_{1}$, the analysis is finished. Otherwise the $\mathcal{\chi}_{\mu_{0}}^{(2)}$ are the second generation of the Lagrangian constraints, which together with $\chi_{\mu}^{(1)}$ define the surface $S_{2}$.

Now it is necessary to study the stability of $\chi_{\mu_{0}}^{(2)}$. It is possible to show that

$$
K \Phi_{\mu_{0}}^{(1)}=\bar{s}_{s_{1}}^{(2)},
$$

where $K$ is the operator defined in (4.6). So the $\chi_{\mu_{0}}^{(2)}$ are associated with the Hamiltonian constraints $\Phi_{\mu_{0}}^{(1)}$. Remembering the splitting (3.17), it follows that we have the following splitting at Lagrangian level:

$$
\begin{array}{lll}
\chi_{\mu_{1}}^{(1)}, & \chi_{\mu_{1}}^{(2)}, & \mu_{1}=1, \ldots, m_{3}, \\
\chi_{\mu_{1}^{\prime}}^{(1)}, & \chi_{\mu_{1}^{\prime}}^{(2)}, & \mu_{1}^{\prime}=1, \ldots, m_{2}-m_{3} .
\end{array}
$$

We see that labeling is compatible with the stability.

Now we can show

$$
\mathbf{F L} *\left\{\Phi_{\mu_{1}^{\prime}}^{(1)}, H_{c}^{(1)}\right\}+v_{v_{1}^{\prime}}(q, \dot{q}) \mathbf{F L} *\left\{\Phi_{\mu_{1}^{(1)}}^{(1)}, \Phi_{\nu_{1}^{\prime}}^{(0)}\right\} \underset{S_{1}}{=} \chi_{\mu_{0}^{\prime}}^{(2)},
$$

$$
\mathrm{FL}^{*} \Phi_{\mu_{1}}^{(2)}{ }_{\bar{S}_{1}} \chi_{\mu_{1}}^{(2)}\left(\bmod \chi_{\mu_{1}^{\prime}}^{(2)}\right)
$$

The proof of (4.26) is more intricate than the first level and is not given here. One can also show

$$
\mathbf{F L} *\left\{\Phi_{\mu_{1}}^{(1)}, H_{c}^{(1)}\right\} \underset{s_{1}}{=} \chi_{\mu_{2}}^{(2)} .
$$

From Eq. (4.26a) we see that the constraints $\chi_{\mu_{1}^{\prime}}^{(2)}$ are not FL-projectable, instead the constraints $\chi_{\mu_{1}}^{(2)}$ are FL-projectable on the surface $S_{1}$, [see Eq. (4.27) ]. We call these objects weakly FL-projectable on $S_{1}$.

Furthermore, using the results of Appendix C we have

$$
\Gamma_{\mu_{0}} \chi_{\mu_{i}^{\prime}}^{(2)}=\mathrm{FL}^{*}\left\{\Phi_{\mu_{\mathrm{i}}^{(1)}}^{(1)}, \Phi_{\mu_{0}}^{(0)}\right\} \underset{S_{i}}{\neq 0}
$$

instead of

$$
\Gamma_{\mu_{0}} \chi_{\mu_{1}}^{(2)}=\mathrm{FL} *\left\{\Phi_{\mu_{1}}^{(1)}, \Phi_{\mu_{0}}^{(0)}\right\} \underset{S_{1}}{=} 0
$$

This analysis suggests that the necessary and sufficient condition for a function $f$ to be weakly FL-projectable on $S_{1}$ is

$$
\Gamma_{\mu_{0}} f \underset{s_{1}}{=} 0, \quad \mu_{0}=1, \ldots, m_{1},
$$

where $\Gamma_{\mu_{0}}$ are the vector vectors fields of Ker FL tangent to $S_{1}$. This result is proved in a separate paper.

Now we need to require the stability of $\chi_{\mu_{0}}^{(2)}$

$$
\begin{gathered}
\frac{d}{d t} \chi_{\mu_{0}}^{(2)} \underset{S_{2}}{=}\left(D^{0}+\beta_{v_{0}^{0}} \Gamma_{v_{0}^{0}}\right) \chi_{\mu_{0}}^{(2)}+\beta_{v_{0}} \Gamma_{v_{0}} \chi_{\mu_{0}}^{(2)} \\
\equiv D^{(1)} \chi_{\mu_{0}}^{(2)}+\beta_{v_{0}} \Gamma_{v_{0}} \chi_{\mu_{0}}^{(2)} .
\end{gathered}
$$

If we consider the stability condition for $\chi_{\mu_{1}^{\prime}}^{(2)}$, we can express the undetermined accelerations $\beta_{v_{1}^{\prime}}$ in terms of the coordinates and velocities:

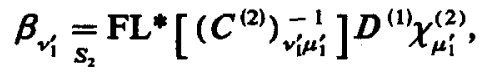

due to the nonsingular character of the matrix $C^{(2)}$, Eq. (3.18). Let us now consider the constraints $\chi_{\mu_{1}}^{(2)}$, we have

$$
\frac{d}{d t} \chi_{\mu_{1}}^{(2)}{ }_{s_{2}} D^{(1)} \chi_{\mu_{1}}^{(2)} \equiv \chi_{\mu_{1}}^{(3)} \text {. }
$$

If the relations $\chi_{\mu_{1}}^{(3)}$, are automatically verified in $S_{2}$ the analysis is finished. Otherwise $\chi_{\mu_{1}}^{(3)}$ are the third generation of the Lagrangian constraints and the procedure continues. At Hamiltonian level we have assumed the existence of a submanifold $M_{f}$ where we can have solutions. This implies that the relations

$$
\left\{\Phi_{\mu_{f}}^{(f)}, H^{(f+1)}\right\} \underset{M_{f}}{=0}
$$

are identities on $M_{f}$. At the Lagrangian level we have

$$
\begin{aligned}
& K \Phi_{\mu_{f}^{\prime}}^{(\cap)} \underset{s_{f}}{=} \chi_{\mu_{f}^{\prime}}^{(f+1)}, \\
& K \Phi_{\mu_{f}}^{(f)}=0,
\end{aligned}
$$

and

$$
\mathbf{F L} *\left\{\Phi_{\mu_{f}^{\prime}}^{(f)}, H_{c}^{(f)}\right\}+v_{v_{f}^{\prime}}(q, \dot{q})\left\{\Phi_{\mu_{f}^{(f)}}^{(f)} \Phi_{\mu_{f}^{\prime}}^{(f)}\right\} \underset{s_{f}}{=} \chi_{\mu_{f}^{f}}^{(f+1)}
$$




$$
\mathbf{F L} *\left\{\Phi_{\mu_{f}}^{(f)} H_{c}^{(f+1)}\right\} \underset{s_{f}}{=} 0
$$

This means that we have no more weakly FL-projectable constraints. However, we have the non-FL-projectable constraints $\chi_{\mu^{\prime}}^{(f+1)}$. If we consider the stability of these constraints, we can obtain the undetermined $\beta_{\mu_{f}^{\prime}}$ acceleration in terms of the coordinate and momenta. At this point the analysis is finished.

Summing up, at every level a (weakly) FL-projectable constraint on a certain submanifold comes from the stability of a Hamiltonian constraint of the preceding level, which is first class with respect to the primary Hamiltonian constraint, while a non-FL-projectable constraint comes from the stability of a Hamiltonian constraint which converts a primary constraint to the second class. Also, if a certain number of velocities are canonically determined at a given level, the same number of accelerations are determined at the next level.

\section{CONCLUSIONS}

The equivalence between the Lagrangian and Hamiltonian formalism for constrained systems has been proved, in the sense that given a solution $q(+)$ of Euler-Lagrange equations of motion, the functions $q(t)$ and $p(t)=\mathscr{P}(q(t)[d q(t) / d t])$ are solutions of the Hamiltonian-Dirac equations of motion and vice versa. Note that neither of these equations is in normal form. This means that we can only have solutions in a submanifold of the respective space. These submanifolds are constructed through an interactive procedure. At the Hamiltonian level, our procedure differs from the standard one. All constraints are classified according to whether or not they are first class with respect to the primary constraints. We have seen that PB matrix of the primary first-class constraints on $\boldsymbol{M}_{\mathbf{0}}$ and the secondary, tertiary, ... constraints are either symmetric or antisymmetric. This implies that our final Hamiltonian $H_{c}^{(f+1)}$ differs from the starred Hamiltonian of Komar and Bergman, but on the final submanifold $M_{f}$ they both yield the same evolution.

At the Lagrangian level, we have seen that the Lagrangian constraints can be obtained from the stability of the Hamiltonian constraints using the $K$ operator (4.6). Furthermore, the Lagrangian constraints that are FL-projectable or weakly FL-projectable are the Lagrangian counterparts of the Hamiltonian constraints, which are first class or second class with respect to the primary Hamiltonian constraints. In fact at every level, a (weakly) FL-projectable constraint on a certain submanifold comes from the stability of a Hamiltonian constraint of the preceding level, which is first class with respect to the primary Hamiltonian constraint, while a non-FL-projectable constraint comes from the stability of a Hamiltonian constraint that converts a primary constraint to the second class. Also, if a certain number of velocities are canonically determined at a given level, the same number of accelerations are determined at the next level.

\section{ACKNOWLEDGMENTS}

We are grateful to Professor Kenneth Rafanelli for a careful reading of the manuscript. One of us (J.G.) would like to acknowledge Professor B. Sakita for the hospitality at the High Energy Group of City College.

\section{APPENDIX A: COMMENTS ON THE INVERSE LEGENDRE TRANSFORMATION}

Here we demonstrate that Eqs. (2.17),

$$
\dot{q}=\frac{\partial H_{c}}{\partial p_{i}}(q, p)+v_{\mu}(q \dot{q}) \frac{\partial \Phi_{\mu}^{(0)}}{\partial p_{i}}(q, p),
$$

for $\dot{q}$ in terms of $q$ and $p$, have solutions only if $(q, p)$ belongs to the submanifold $\boldsymbol{M}_{0}$ of the primary constraints. We know that when $(q, p)$ belongs to $\boldsymbol{M}_{0}$, the solutions are given by (2.16). Therefore, we have the identities

$$
\dot{q}^{i} \equiv \mathrm{FL}^{*} \frac{\partial H_{c}}{\partial p_{i}}+v_{\mu}(q \dot{q}) \mathrm{FL}^{*} \frac{\partial \Phi_{\mu}^{(0)}}{\partial p_{i}} .
$$

Now at fixed $q$ we consider an infinitesimal displacement $p+d p$ from a point $(q, p) \in M_{0}$. We want to know if there exists a solution of (A1), $\dot{q}+d \dot{q}$, derived continuously from the solution, $\dot{q}$, with data $(q, p) \in M_{0}$. If such a solution exists, the following identities must be verified:

$$
\begin{aligned}
d \dot{q}^{i}= & \mathrm{FL}^{*} \frac{\partial^{2} H_{c}}{\partial p_{i} \partial p_{j}} d p_{j}+\frac{\partial v_{\mu}(q, \dot{q})}{\partial \dot{q}^{j}} \mathrm{FL}^{*} \frac{\partial \Phi_{\mu}^{(0)}}{\partial p_{i}} d \dot{q}^{j} \\
& +v_{\mu}(q, \dot{q}) \mathrm{FL}^{*} \frac{\partial^{2} \Phi_{\mu}^{(0)}}{\partial p_{i} \partial p_{j}} d p_{j} .
\end{aligned}
$$

In order to study when these identities are verified, let us consider the completeness relation

$$
\delta_{j}^{i}=\tilde{\gamma}_{\mu j} \gamma_{\mu}^{i}+M^{i k} W_{k j},
$$

where

$$
\begin{aligned}
\tilde{\gamma}_{\mu j} & =\frac{\partial v_{\mu}(q, \dot{q})}{\partial \dot{q}^{j}}, \quad \gamma_{\mu}^{i}=\mathrm{FL}^{*} \frac{\partial \Phi_{\mu}^{(0)}}{\partial p_{i}}, \\
M^{i k} & =\mathrm{FL}^{*} \frac{\partial^{2} H_{c}}{\partial p_{i} \partial p_{k}}+v_{\mu}(q, \dot{q}) \mathrm{FL}^{*} \frac{\partial^{2} \Phi_{\mu}}{\partial p_{i} \partial p}, \\
W_{k j} & =\frac{\partial^{2} L}{\partial \dot{q}_{k} \partial q_{j}} .
\end{aligned}
$$

Equation (A4) can be obtained by taking the derivative of (A2) with respect to $q_{j}$. Note that $M^{i k}$ is not unambiguously defined in $\Lambda^{\circ}\left(T^{*} Q\right)$. This is due to the ambiguity of the definition of $H_{c}$ out of the surface $M_{0}$.

The change

$$
H_{c}(q, p) \rightarrow H_{c}^{\prime}(q, p)=H_{c}(q, p)+\lambda_{\mu}(q, p) \Phi_{\mu}^{(0)}(q, p)
$$

with $\lambda_{\mu}$ arbitrary describes the arbitrariness of the Hamiltonian. This change produces a new definition of the functions $v_{\mu}$ of (2.17),

$$
v_{\mu}^{\prime}(q, \dot{q})=v_{\mu}(q, \dot{q})-\mathrm{FL}^{*} \lambda_{\mu},
$$

and consequently a change in the matrices $M^{i k}$,

$$
M^{\prime i k}=M^{i k}+\mathrm{FL}^{*} \frac{\partial \lambda_{\mu}}{\partial p_{i}} \gamma_{\mu}^{k}+\mathrm{FL}^{*} \frac{\partial \lambda_{\mu}}{\partial p_{k}} \gamma_{\mu}^{\prime} .
$$

Equation (A3) can be written [using (A4) and (A5)] as 


$$
M^{i k}\left(W_{k j} d \dot{q}^{j}-d \mathrm{FL}^{*} p_{k}\right)=0,
$$

where $M^{i k}$ is any element of the family (A7). Since in that family there always exists a nonsingular matrix, Eq. (A8) is verified only if

$$
W_{k j} d \dot{q}^{j}=d \mathrm{FL}^{*} p_{k} .
$$

The necessary and sufficient condition for (A9) to be fulfilled is that

$$
\gamma_{\mu}^{k} d \mathrm{FL}^{*} p_{k}=0
$$

where $\gamma_{\mu}^{k}$ are the null vectors of $W$, Eq. (A5). Therefore, we have

$$
\mathrm{FL}^{*} d \Phi_{\mu}=0,
$$

and therefore we conclude that the only displacement $p \rightarrow p+d p$ that allow changes on the solutions $\dot{q} \rightarrow \dot{q}+d \dot{q}$ are those made on the surface $M_{0}$.

\section{APPENDIX B: STRUCTURE OF THE CONSTRAINTS POISSON BRACKET}

In Sec. III we stated that the matrix of PB between a subset of primary first-class constraints on $M_{0}$ and the secondary, tertiary, ... constraints was either symmetric or antisymmetric. Here we demonstrate this property explicitly. Let us begin with the matrix of PB between the primary constraints $\Phi_{\mu_{0}}^{(0)}$ and the secondary constraints $\Phi_{\mu_{0}}^{(1)}$. Taking into account the definition of $\Phi_{\mu_{0}}^{(1)}$, the Jacobi identity, and the first-class character of $\Phi_{\mu_{0}}^{(0)}$ on $M_{0}$ we have

$$
\begin{aligned}
\left\{\Phi_{\mu_{0}}^{(1)}, \Phi_{v_{0}}^{(0)}\right\} & =\left\{\left\{\Phi_{\mu_{0}}^{(0)}, H_{c}^{(1)}\right\}, \Phi_{v_{0}}^{(0)}\right\}=\left\{\left\{\Phi_{\mu_{0}}^{(0)}, H_{c}\right\}, \Phi_{v_{0}}^{(0)}\right\} \\
& =-\left\{\left\{\Phi_{\nu_{0}}^{(0)}, \Phi_{\mu_{0}}^{(0)}\right\}, H_{c}\right\}-\left\{\left\{H_{c}, \Phi_{v_{0}}^{(0)}\right\}, \Phi_{\mu_{0}}^{(0)}\right\} \\
& =\left\{\Phi_{v_{0}}^{(1)}, \Phi_{\mu_{0}}^{(0)}\right\}-\left\{\left\{\Phi_{v_{0}}^{(0)}, \Phi_{\mu_{0}}^{(0)}\right\}, H_{c}\right\} .
\end{aligned}
$$

Consider the last term of Eq. (B1), since the $\Phi_{\mu_{0}}^{(0)}$ 's are first class on $M_{0}$ then

$$
\left\{\left\{\Phi_{\mu_{0}}^{(0)}, \Phi_{\nu_{0}}^{(0)}\right\} \Phi_{v_{0}^{\prime}}^{(0)}\right\} \underset{M_{0}}{=} 0
$$

this implies that

$$
\left\{\Phi_{\mu_{0}}, \Phi_{v_{0}}^{(0)}\right\}=O\left(\Phi_{\mu_{0}}^{(0)}\right)+O^{2}\left(\Phi_{\mu_{0}^{\prime}}^{(0)}\right),
$$

where $O\left(\Phi_{\mu_{0}}^{(0)}\right)$ is a function that contains a term linear in $\Phi_{\mu_{0}}^{(0)}$, and $O^{2}\left(\Phi_{\mu_{0}^{\prime}}^{(0)}\right)$ is a function of $\Phi_{\mu_{0}}^{(0)}$ that contains a term quadratic in $\Phi_{\mu_{0}^{\prime}}^{(0)}$ as the lowest-order term. This means that the last term of Eq. (B1) vanishes on the surface $M_{1}$, therefore (B1) becomes

$$
\left\{\Phi_{\mu_{0}}^{(1)}, \Phi_{v_{0}}^{(0)}\right\} \underset{M_{1}}{=}\left\{\Phi_{\nu_{0}}^{(1)}, \Phi_{\mu_{0}}^{(0)}\right\} .
$$

This means that the above matrix is symmetric with respect to the interchange of $\mu_{0}$ and $v_{0}$ on $M_{1}$.

Let us now consider the matrix of the PB between the primary first class constraints on $M_{2}$ and the tertiary constraints. We have

$$
\begin{aligned}
\left\{\Phi_{\mu_{1}}^{(2)}, \Phi_{\nu_{1}}^{(0)}\right\} & =\left\{\left\{\Phi_{\mu_{1}}^{(1)}, H_{c}^{(2)}\right\} \Phi_{v_{1}}^{(0)}\right\}=\underset{M_{1}}{=}\left\{\left\{\Phi_{\mu_{1}}^{(1)}, H_{c}^{(1)}\right\} \Phi_{v_{1}}^{(0)}\right\} \\
& =-\left\{\left\{\Phi_{v_{1}}^{(0)}, \Phi_{\mu_{1}}^{(1)}\right\} H_{c}^{(1)}\right\}+\left\{\Phi_{v_{1}}^{(1)}, \Phi_{\mu_{1}}^{(1)}\right\}
\end{aligned}
$$

$$
\begin{aligned}
= & -\left\{\left\{\Phi_{\nu_{1}}^{(0)}, \Phi_{\mu_{1}}^{(1)}\right\} H_{c}^{(1)}\right\} \\
& -\left\{H_{c}^{(1)}\left\{\Phi_{\nu_{1}}^{(1)}, \Phi_{\mu_{1}}^{(0)}\right\}\right\}-\left\{\Phi_{\mu_{1}}^{(0)}\left\{H_{c}^{(1)}, \Phi_{\nu_{1}}^{(1)}\right\}\right\} \\
= & -\left\{\left\{\Phi_{\nu_{1}}^{(0)}, \Phi_{\mu_{1}}^{(1)}\right\} H_{c}^{(1)}\right\} \\
& +\left\{\left\{\Phi_{\nu_{1}}^{(1)}, \Phi_{\mu_{1}}^{(0)}\right\} H_{c}^{(1)}\right\}-\left\{\Phi_{\nu_{1}}^{(2)}, \Phi_{\mu_{1}}^{(0)}\right\} .
\end{aligned}
$$

The PB $\left\{\Phi_{\mu_{1}}^{(0)}, \Phi_{v_{1}}^{(1)}\right\}$ vanishes on $M_{1}$, furthermore we have $\left\{\left\{\Phi_{\mu_{1}}^{(0)}, \Phi_{\nu_{1}}^{(1)}\right\} \Phi_{\mu_{1}}^{(1)}\right\}$

$$
=-\left\{\left\{\Phi_{\mu_{1}^{\prime}}^{(0)}, \Phi_{\mu_{1}}^{(0)}\right\} \Phi_{v_{1}}^{(1)}\right\}-\left\{\left\{\Phi_{v_{1}}^{(1)} \Phi_{\mu_{1}^{\prime}}^{(0)}\right\} \Phi_{\mu_{1}}^{(0)}\right\} \underset{M_{1}}{=} 0,
$$

therefore

$\left\{\Phi_{\mu_{2}}^{(0)}, \Phi_{\nu_{1}}^{(1)}\right\}=O\left(\Phi_{\mu_{0}^{\prime}}^{(0)}+O\left(\Phi_{\mu_{1}^{\prime}}^{(0)}\right)+O\left(\Phi_{\mu_{1}}^{(0)}\right)+O^{2}\left(\Phi_{\mu_{1}^{\prime}}^{(0)}\right)\right.$, which implies

$$
\left\{\left\{\Phi_{v_{1}}^{(0)}, \Phi_{\mu_{1}}^{(1)}\right\} H_{c}^{(1)}\right\} \underset{M_{2}}{=} 0 .
$$

Equation (B5) becomes

$$
\left\{\Phi_{\mu_{1}}^{(2)}, \Phi_{v_{1}}^{(0)}\right\}_{M_{2}}-\left\{\Phi_{v_{1}}^{(2)}, \Phi_{\mu_{1}}^{(0)}\right\}
$$

which means that this matrix of PB's is antisymmetric. This antisymmetric property is due to the twofold application of the Jacobi identity. In the general case for the primary first class constraints on $M_{k}$ and the $\mathrm{K}$-ary constraints, we will have

$$
\left\{\Phi_{\mu_{k}}^{(k)}, \Phi_{\nu_{k}}^{(0)}\right\} \underset{M_{k}}{=}(-1)^{k+1}\left\{\Phi_{\nu_{k}}^{(k)}, \Phi_{\mu_{k}}^{(0)}\right\}
$$

\section{APPENDIX C: RELATION TO DIRAC BRACKET}

We want to study the relation between the procedure developed in the text and the standard Dirac bracket formalism for the second class constraints when there are no quartiary constraints. In this case the second class constraints are $\Phi_{\mu_{0}^{\prime}}^{(0)}, \Phi_{\mu_{1}^{\prime}}^{(0)}, \Phi_{\mu_{2}^{\prime}}^{(0)}, \Phi_{\mu_{1}^{\prime}}^{(1)}, \Phi_{\mu_{2}^{\prime}}^{(1)}, \Phi_{\mu_{2}^{\prime}}^{(2)}$, if we use the Dirac bracket with respect to $\Phi_{\mu_{0}^{\prime}}^{(0)} \Phi_{\mu_{1}^{\prime}}^{(0)} \Phi_{\mu_{1}^{\prime}}^{(1)}$, Eq. (3.48), we only need to consider the constraints $\Phi_{\mu_{2}^{\prime}}^{(0)}, \Phi_{\mu_{2}^{\prime}}^{(1)}, \Phi_{\mu_{2}^{\prime}}^{(2)}$. Let $\chi_{\mu_{2}^{\prime}}$ denote anyone of those constraints and matrix $D_{\mu_{2}^{\prime} v_{2}^{\prime}}^{(3)} \equiv\left\{\chi_{\mu_{2}^{\prime}} \chi_{v_{2}^{\prime}}\right\}^{H_{2}}$. Using the relations

$$
\left\{\Phi_{\mu_{2}^{\prime}}^{(0)}, \Phi_{v_{2}^{\prime}}^{(2)}\right\}^{H_{2}}=\left\{\Phi_{M_{1}}^{(0)}, \Phi_{\nu_{2}}^{(2)}\right\},
$$

we have

$$
D^{(3)}=\left(\begin{array}{ccc}
0 & 0 & -C^{(3)} \\
0 & -C_{1}^{(3)} & -B \\
C^{(3)} & B & Q
\end{array}\right)
$$

where $C^{(3)}$ is given by Eq. (3.32). The inverse matrix $D^{(3)-1}$ is given by

$$
D^{(3)-1}=\left(\begin{array}{ccc}
M & C^{(3)-1} B C^{(3)-1} & C^{(3)-1} \\
-C^{(3)-1} B C^{(3)-1} & -C^{(3)-1} & 0 \\
-C^{(3)-1} & 0 & 0
\end{array}\right),
$$

where

$$
M=C^{(3)-1} Q C^{(3)-1}+C^{(3)-1} B C^{(3)-1} B C^{(3)-1} .
$$


At this point we can write the final Dirac bracket

$$
\{A, B\}^{H_{3}}=\{A, B\}^{H_{2}}-\left\{A, \chi_{\mu_{2}^{\prime}}\right\}^{H_{2}} D_{\mu_{2}^{\prime} \nu_{2}^{\prime}}^{(3)}\left\{\chi_{v_{2}^{\prime}} B\right\}^{H_{2}}
$$

If we consider ( $\mathrm{C5}$ ) for the case $B=H_{c}$, we obtain

$$
\left\{A, H_{c}\right\}^{H_{3}}=\left\{A, H_{c}^{(3)}\right\} .
$$

Therefore the evolution on $M_{2}$ with the Dirac bracket formalism coincides with our procedure.

\section{APPENDIX D: PROPERTIES OF THE OPERATOR $K$}

Here we want to show that the operator $K$,

$$
K: \Lambda^{0}\left(T^{*} Q\right) \rightarrow \Lambda^{0}(T Q),
$$

given by

$$
K=\dot{q}^{i} \mathrm{FL} * \frac{\partial}{\partial q^{i}}+\frac{\partial L}{\partial q^{i}} \mathrm{FL}^{*} \frac{\partial}{\partial p_{i}},
$$

applied to the primary Hamiltonian constraints $\Phi_{\mu}^{(0)}$ produces the first generation of Lagrangian constraints $\chi_{\mu}^{(0)}$. In fact

$$
K \Phi_{\mu}^{(0)}=\dot{q}^{i} \mathrm{FL}^{*} \frac{\partial \Phi_{\mu}^{(0)}}{\partial q^{i}}+\frac{\partial L}{\partial q^{i}} \mathrm{FL} * \frac{\partial \Phi_{\mu}^{(0)}}{\partial p_{i}}, \quad \mu=1, \ldots, m_{1},
$$

and from Eqs. (2.7) and (2.21) we have

$$
\begin{aligned}
& \mathrm{FL}^{*} \frac{\partial \Phi_{\mu}^{(0)}}{\partial p_{i}}=\gamma_{\mu}^{i}, \\
& \mathrm{FL}^{*} \frac{\partial \Phi_{\mu}^{(0)}}{\partial q^{i}}=-\gamma_{\mu}^{j} \frac{\partial^{2} L}{\partial q^{j} \partial q^{i}}=-\Gamma_{\mu} \frac{\partial L}{\partial q^{i}},
\end{aligned}
$$

therefore

$$
K \Phi_{\mu}^{(0)}=\gamma_{\mu}^{i}\left(\frac{\partial L}{\partial q^{i}}-\frac{\partial^{2} L}{\partial \dot{q}^{i} \partial q^{j}} \dot{q}^{j}\right)=\chi_{\mu}^{(1)} .
$$

In general, the operator $K$ applied to a function $g \in \Lambda^{0}\left(T^{*} Q\right)$ gives its temporal derivative expressed in $\Lambda^{0}(T Q)$, which we denote by $f_{g}(q, \dot{q})$. Let us now study the projectability of $f_{g}$ :

$$
\left(\Gamma_{\mu} f_{g}\right)=\left(\mathrm{FL}^{*} \frac{\partial g}{\partial q^{i}}\right) \Gamma_{\mu} q^{i}+\left(\mathrm{FL} * \frac{\partial g}{\partial q^{i}}\right) \Gamma_{\mu} \frac{\partial L}{\partial q^{i}} .
$$

Using (D4) and (D5) we obtain

$$
\Gamma_{\mu} f_{g}=\mathbf{F L} *\left\{g, \Phi_{\mu}^{(0)}\right\}
$$

Therefore $f_{g} \leftarrow \Lambda^{0}(T Q)$ will be FL-projectable if $g \leftarrow \Lambda^{0}(T Q)$ is a first class function with respect to the primary first class constraints on $\boldsymbol{M}_{0}$. In particular using (D8) we have

$$
\Gamma_{\mu} \chi_{\nu}^{(1)}=\mathrm{FL}^{*}\left\{\Phi_{v}^{(0)}, \Phi_{\mu}^{(0)}\right\},
$$

which states only that the Lagrangian constraints associated with the first class primary constraints are FL-projectable. Another consequence of (D8) is

$$
\Gamma_{\mu_{0}} \mathcal{X}_{v_{0}}^{(2)}=\mathrm{FL} *\left\{\Phi_{v_{0}}^{(1)}, \Phi_{\mu_{0}}^{(0)}\right\},
$$

which tells us that the only Lagrangian constraints associated with the secondary Hamiltonian constraints which are first class with respect to the primary first-class constraints on $M_{0}$ are weakly FL-projectable.

'P. A. M. Dirac, Can. J. Math. 2, 129 (1950); Proc. R. Soc. London Ser. A 246, 326 (1958).

${ }^{2}$ J. L. Anderson and P. G. Bergmann, Phys. Rev. 83, 1018 (1951); P. G. Bergmann and I. Goldberg, Phys. Rev. 98, 531 (1955).

${ }^{3}$ It is impossible to quote here all relevant contributions. Please see references to the original literature in the quoted review papers.

${ }^{4}$ E. C. G. Sudarshan and N. Mukunda, Classical Dynamics: $A$ Modern Perspective (Wiley, New York, 1974).

${ }^{5} \mathrm{~K}$. Sundermeyer, "Constrained dynamics," in Lecture Notes in Physics, Vol. 169 (Springer, Berlin, 1982).

'See, for example, D. Dominici, G. Longhi, J. Gomis and J. M. Pons, J. Math. Phys. 25, 2439 (1984).

${ }^{7}$ G. Marmo, N. Mukunda, and J. Samuel, Riv. Nuovo Cimento 6, 4 (1983)

8J. Llosa (Editor), "Relativistic action at a distance: Classical and quantum aspects," Lecture Notes in Physics, Vol. 162, edited by J. Llosa (Springer, Berlin, 1982).

${ }^{9}$ H. Sazdjian, Phys. Lett. B 156, 381 (1985).

${ }^{10}$ M. Gotay and J. M. Nester, Ann. Inst. H. Poincarè 30, 129 (1979).

"K. Kamimura, Nuovo Cimento B 69, 33 (1982).

${ }^{12}$ P. G. Bergmann and A. Komar, Phys. Rev. Lett. 4, 432 (1960).

${ }^{13}$ R. Skinner and R. Rusk, J. Math. Phys. 24, 2589 (1983). 